\title{
Avian Influenza Infection in Humans
}

\author{
Dr. O. M. Sogaolu \\ Pulmonology Unit, Department of Medicine, \\ University College Hospital, Ibadan.
}

\section{INTRODUCTION}

Avian influenza is an infection caused by avian (bird) influenza (Flu) viruses. These influenza viruses occur naturally among birds. Wild birds worldwide carry the viruses in their intestines, but usually do not get sick from them[1].

It is a highly contagious viral infection which can affect all species of birds and can manifest itself in different ways depending mainly on the pathogenicity of the virus involved and on the species affected[2].

Avian influenza strains in domestic chickens and turkeys are classified according to disease severity, with two recognized forms highly pathogenic avian influenza (HPAl) also known as fowl plaque, and low-pathogenic avian influenza (LPAI). Avian influenza viruses that cause HPAI are highly virulent and mortality rates in infected flocks often approach $100 \%$. LPAI viruses are generally of lower virulence, but these viruses can serve as progenitors to HPAI viruses. The current strain of $\mathrm{H} 5 \mathrm{~N} 1$ responsible for die-offs of domestic birds in Asia is an HPAI strain. All HPAI strains identified to date have involved $\mathrm{H} 5$ and $\mathrm{H} 7$ subtype $[1,3,4,5]$.

Infected birds shed influenza virus in their saliva, nasal secretions and faeces. Susceptible birds become infected when they have contact with contaminated secretions or excretions or with surfaces that are contaminated with secretions or excretions

All Correspondence to Dr. O. M. Sogaolu

Pulmonology Unit,

Department of Medicine,

University College Hospital,

P.M.B. 5116, Ibadan

E-Mail: sogee2002@yahoo.com from infected birds. Domesticated birds may become infected with avian influenza virus through direct contact with infected waterfowl or other infected poultry, or through contact with surfaces (such as dirt or cages) or materials (such as water or $r$ ed) that have been contaminated with the virus[2].

\section{INFLUENZÄ A VIRUS AND HUMAN DISEASE}

Avian influenza is caused by influenza A virus. Influenza A viruses are a major cause of influenza in humans. It belongs to the family orthomyxoviridae. It is an enveloped virus consisting of spike-shaped surface proteins, and matrix (M) proteins surrounding a helical segmented nucleocapsid ( 6 to $8 \mathrm{seg}$ ments) $[1,4,6]$.

The virus envelope glycoproteins (haemaglutinins HA and neuraminidase NA) are distributed evenly over the virion surface, forming characteristics spike-shaped structures. Antigenic variation in these proteins is used as part of the influenza A virus subtype definition (but not used for influenza $\mathrm{B}$ or $\mathrm{C}$ viruses). There are 16 different $\mathrm{HA}$ antigens (H1 to H16) and nine differe it NA antigens (N1 to N9) for influenza A [1].

All past influenza pandemic have been caused by influenza $A$ viruses. Human disease historically has been caused by three subtypes of $\mathrm{HA}(\mathrm{H} 1, \mathrm{H} 2$ and $\mathrm{H} 3$ ) and two subtypes of NA (N1 and N2). More recently human disease has been recognized to be caused by additional $H A$ subtypes including $\mathrm{H} 5, \mathrm{H} 7$ and H9. All known subtypes of influenza $\mathrm{A}$ can be found in bird, and feral aquatic birds are the major reservoir for influenza $A$ viruses $[1,4]$. 
Usually, avian influenza virus refers to influenza A virus found chiefly in birds, but infections with these viruses can occur humans. The risk from avian influenza is generally low to most people, because the viruses do not usually infect humans.

However, confirmed cases of human infection from several subtypes of avian influenza infection have been reported since 1997. Most cases of avian influenza infection in human have resulted from contact with infected poultry (e.g., domesticated chicken, ducks and turkeys) or surfaces contaminated with secretion/excretions srom infected birds $[1,3,4]$.

\section{AVIAN INFLUENZA A (H5N1) OUTBREAKS}

Of the few avian influenza viruses that have crossed the species barrier to infect humans, H5N1 has caused the largest number of detected cases of severe disease and death in humans. In the current outbreaks in Asia and Europe more than half of those infected with the virus have died $[1,3]$.

Most cases have occurred in previously healthy children and young adults. The spread of $\mathrm{H} 5 \mathrm{~N} 1$ virus from person to person has been limited and has not continued beyond one person. Nonetheless, because all influenza viruses have the ability to change, scientists are concerned that $\mathrm{H} 5 \mathrm{~N} 1$ virus one day could be able to infect humans and spread easily from one person to another. Because these viruses do not commo. ly infect human, there is little or no immune protection against them in the human population[1,5].

\section{Avian Influenza in Humans: Epidemiologic Considerations}

WHO has officially recognized more than $175 \mathrm{hu}-$ man cases of $\mathrm{H} 5 \mathrm{~N} 1$ influenza; cases have been reported from Vietnam, Thailand, Cambodia, Indonesia, China, Turkey and Iraq. The case-fatality rate is about $50 \%[3,4]$.

Low perceived risk and high population exposures to live chicken appear to be factors that are contributing to the spread of H5N1 in Asia[6] Most recognized human cases have involved direct contact with poultry. . The types of exposures that have been identified to date include:
- Plucking and preparing diseased birds

- Handling fighting cocks

- Playing with poultry (particularly symptomatic ducks)

- Consumption of duck blood and possibly undercooked poultry.

Given the survival of influenza $A(H 5 N 1)$ in the environment, (viable at moderate temperatures for long periods in the environment and can survive indefinitely in frozen material. It can survive for 4 days in water at $22^{\circ} \mathrm{C}$ and for over 30 days at $0^{\circ} \mathrm{C}$ ) several other modes of transmission are theoretically possible. Oral ingestion of contaminated water during swimming and direct intranasal or conjunctival inoculation during exposure to water are other potential modes, as is contamination of hands from infected fomites and subsequent self-inoculation. The widespread use of untreated poultry faeces as fertilizer is another possible risk factor $[4,7,8]$.

\section{Clinical Features}

The incubation period for most patients with $\mathrm{H} 5 \mathrm{~N} 1$ influenza is $2-4$ days; $[8]$ however, the range appears to be as long as 8 days $[1,4,9]$. The case to case intervals in household clusters have generally been about 2-5, days but the upper limit has been 8 to 17 days, possibly owing to unrecognized exposure to infected animals or environmental sources. $[4,10,11]$

The clinical spectrum of influenza $A$ in humans is based on descriptions of hospitalized patients. Most patients have initial symptoms of high fever $\left(>3^{\circ} \mathrm{C}\right)$ and an influenza- like illness with lower respiratory tract symptoms $[1,8]$. Clinical presentations for different groups of patients in Asia reported include; headache, myalgia, diarrhoea, abdominal pain, vomiting conjunctivitis. Presence of pulmonary infiltrates lymphopenia, thrombocytopenia, increased aminotransferase levels, and development of respiratory failure (usually with ARDS) $[1,8,9,10,11]$.

Watery diarrhea without blood or inflammatory changes appears to be more common than in influenza due to human viruses and may precederespiratory manifestations by up to one week[8]. One report describes two patients who presented with an encephalopathic illness and diarrhea without apparent respiratory symptoms [12]. 


\section{LABORATORY TESTING FOR INFLU- ENZA IN HUMAN}

Laboratory tests for influenza include - viral culture, polymerase chain reaction (PCR), rapid antigen testing and immunofluorescence $[14,15]$. Laboratory tests are required for specific identification of avian influenza. The most likely ways that an avian influenza strain would be detected in the human population are:

(1) Outbreak investigations or investigation of unexplained death in a previously healthy individual.

(2) Influenza surveillance with laboratory testing.

(3) Investigation of unusual laboratory findings.

\section{Specimen Collection}

Appropriate specimen for testing includes:

Respiratory specimen
(a) nasopharyngeal wash / aspirate
(b) nasopharyngeal swab/throat swab
(c) Bronchoalveolar lavage
(d) Tracheal aspirate
(e) Pleural fluid tap
(f) Sputum.
(g) Central (hilar) lung with segmental bron chi
(h) Right and left primary bronchi
(i) Trachea (proximal and distal)
(j) Lung parenchyma tissue

Blood - Serum for antibody testing (serology usually for retrospective diagnosis of infection).

Autopsy specimen for immunohistochemistry (IHC) staining $[1,15]$.

\section{Case Detection}

The possibility of influenza A (H5N1) should be considered in the following [4]:

Ø All patients with severe acute respiratory illness in countries or territories with animal influenza A (H5N1). Particularly in patients who have been exposed to poultry.

Ø Patients presenting with serious unexplained illness (e.g. encephalopathy or diarrhea) in areas with known influenza A (H5N1) activity in human or animals.

\section{Pathological Findings}

Limited postmortem analyses have documented severe pulmonary injury with histopathological changes of diffuse alveolar damage. Changes include filling of the alveolar spaces with fibrinous exudates and red cells, hyaline- membrane formation, vascular congestion, infiltration of lymphocytes into the interstitial areas, and the proliferation of reactive fibroblast[16].

Antemortem biopsy of bone marrow specimens has shown reactive histiocytosis with haemphagocytosis in several patients and lymphoid depletion and atypical lymp' ocytes have been noted in spleen and lymphoid tissues at autopsy[ $[10,11]$.

\section{Hospitalization}

Patients with suspected or proven influenza $A$ infection should be hospitalized in isolation for clinical monitoring, appropriate diagnostic testing and antiviral therapy. Supportive care with provision of supplemental oxygen and ventilatory support is the foundation of management $[1,4]$.

\section{Antiviral Agents}

Patients with suspected influenza A (H5N1) should promptly receive a neuraminidase inhibitor (NIs; Oseltamivir [Tamiflu] and Zanamivir [Relenza] [17].

NIs can reduce the duration of illness for both influenza $A$ and $B$ if given early in the clinical course (i.e. within 48 hours after illness onset). Oseltamivir is approved for treatment of influenza in adults and children more than 1 year of age and Zanamivir (Relenza) is approved for treatments of adults and children older than 7years [18].

The H5N1 virus that has caused human illness and death in Asia is resistant to amantadine and Rimantadine, two antiviral medications commonly used for influenza [1].

\section{PREVENTION}

\section{Vaccine Development (Immunization)}

There is currently no commercially available vaccine to protect human against H5N1 virus. (1) However vaccine development efforts are taking place 
particularly because of concerns about the pandemic potential of $\mathrm{H} 5 \mathrm{~N} 1$ influenza virus. Clinical trials of candidate $\mathrm{H} 5 \mathrm{~N} 1$ vaccines are currently under way. [19].

\section{Infection Control Guidelines for H5N1 Avian} Influenza

In May 2004, CDC and WHO issued infection control guidelines for prevention of transmission of $\mathrm{H} 5 \mathrm{~N} 1$ influenzain healthcare settings. Summaries from CDC and WHO of recommended isolation precautions are outlined in the table below.(20-22). Both agencies recommend that Airborne Precautions be implemented, if possible.

Isolation Precautions for Patients With

H5N1 Avian Influenza

CDC Recommendations

Standard Precautions

Pay careful attention to hand hygiene before and after all patient contact or contact with items potentially contaminated with respiratory secretions.

\section{Contact Precautions}

Use gloves and gown during all patient contact.

Use dedicated equipment such as stethoscopes, disposable blood pressure cuffs, and disposable thermometers.

Eye protection (ie, goggles or face shields)

Wear when within $3 \mathrm{ft}$ of patient.

\section{Airborne Precautions}

Place patient in an AI'. Such rooms should have monitored negative air pressure in relation to corridor, with 6 to $12 \mathrm{ACH}$, and should exhaust air directly outside or have recirculated air filtered by a HEPA filter. If an AIR is unavailable, contact the healthcare facility engineer to assist or use portable HEPA filters to augment ACH. Use a fit-tested respirator, at least as protective as a NIOSH-approved N95 filtering facepiece (i.e., disposable) respirator, when entering room.

\section{WHO Recommendations}

Standard Precautions

Droplet Precautions

\section{Contact Precautions}

Airborne Precautions (including use of highefficiency masks and negative-pressure rooms when available)

\section{Abbreviations:}

$\mathrm{ACH}=$ air changes per hour;

$\mathrm{AIR}=$ airborne isolation room;

HEPA = high-efficiency particulate air;

NIOSH = National Institute of Occupational Safety and Health.

\section{House hold and close contact}

Household contacts of persons with confirmed cases of influenza A (H5N1) should receive post exposure prophylaxis with use of $75 \mathrm{mg}$ of oseltamivir once daily for 7 to 10 days.

Contacts of a patient with proven or suspected virus should monitor their temperature and symptoms . For others who have had an unprotected exposure to an infected person or to an environmental source (e.g exposure to poultry) implicated in the transmission of influenza $A(\mathrm{H} 5 \mathrm{~N} 1)$, post exposure chemoprophylaxis as described above may be indicated $[23,24]$.

\section{Food safety issues}

In November 2005, WHO [25] issued a statement on food safety issue. This statement includes the following amongst others:

- The H5N1 avian influenza virus is not transmitted to humans through properly cooked food. The virus is sensitive to heat and normal temperature used for cooking (so that food reaches $70^{\circ} \mathrm{C}$ in all parts) will kill the virus.

- To date, no evidence indicates that any person has become infected with the H5N1 virus following the consumption of properly 
cooked poultry products, even in cases where the food item contained the virus prior to cooking.

- In countries with outbreaks, thorough cooking is imperative. Consumers need to be sure that all parts of the poultry are fully cooked (no "pink" parts) and that eggs, too are properly cooked (no "runny" yolks).

\section{CONCLUSIONS}

Humans remain rarely affected by avian influenza and if they are, the disease in humans is often mild and transitory. However, human infections with HPAI viruses may be even fatal. Therefore, great attention is required to be given to the disease and several actions are constantly taken in the world in order to avoid new outbreaks and eradicate as soon as possible any new cases in poultry or other birds.

WHO continues to issue and update travel recommendations consistent with Phase 3 of the sixphase pandemic alert [26]. No one can predict when a pandemic might occur. Thus there is need for all to be alert and remain aware of the on-going epidemic, bearing in mind the increasing threat to world health from emerging and re-emerging diseases [27].

\section{REFERENCES}

1. CDC. Outbreak notice: Update: Human infection with avian influenza $A$ (H5N1 virus in Asia. Released Sep 23, 2005; updated frequently.

2. Europa - Animal Health and Welfare http/www accessed on

3. World Health Organisation. WHO Interim guidelines on clinical management of humans infected by influenza A (H5N1), February 20, 2004. (Accessed www.who.int/csr/disease/avian_influenza/ guidelines; February 20, 2004.

4. WHO Writing Committee of WHO Consultation on Human Influenza A/H5. Avian influenza A (H5N1) infection in humans. N Eng J Med 2005; 353: 1374-1385

5. Liu J, Xiao H, Lei F, et al. Highly pathogenic $\mathrm{H} 5 \mathrm{~N} 1$ influenza virus infection in migratory birds. Science 2005; 309: 1206

6. Chen H, Smith JD, Zhang SY, et al. Avian flu: $\mathrm{H} 5 \mathrm{~N} 1$ virus outbreak in migratory waterfowl. Nature 2005; 436: 191-192.
7. Fielding R, Lam WTW, Ho EYY, et al. Avian influenza risk perceptionk Hong Kong. Emerg Infect Dis 2005 May; 11(5): 677-682

8. Liem NT, World Health Organisation International Avian Influenza Investigation Team, Vietnam, Lim W. Lack of H5N1 avian influenza transmission to hospital. Employees. Hanoi, 2004. Emerg Infect Dis 2005; 11:210-215

9. Apisarnthanarak A, Kirphati R, Thongphubeth K et al. Atypical influenza (H5N1). Emerg Infect Dis 2004; 10: 1321-1324

10. Yuen KY, Chan PK, Peiris M, et al. Clinical features and rapid viral diagnosis of human disease associated with avian influenza $A H 5 N 1$ virus. Lancet 1998; 351:467- 471

11. Chorpitayasmondh T, Ungehkusak $K$, Hanshaoworakul W, et al. Human disease from influenza A (H5N1), Emerg Infect Dis 2005; 11:201209

12. Hien TT, Liem NT, Dung NT, et al. Avian influenza $\mathrm{A}(\mathrm{H} 5 \mathrm{~N} 1)$ in 10 patients in Vietnam. $\mathrm{N}$ Eng J Med 2004; 350: 1179-1188

13. de Jong MD, Cam BV, Qui PT, et al. Fatal Avian Influenza A(H5N1) in a child presenting with diarrhea followed by coma. N Eng J Med 2005; 352: 686-691

14. Peiris JS, Yu WC, Leung CW, et al. Re-emergence of fatal human influenza A sub-type H5N1 disease. Lancet 2004; 363: 617-619

15. World Health Organization. Recommended laboratory tests to identify influenza $\mathrm{A} / \mathrm{H} 5$ virus in specimens from patients with an influenza-like illness. 2005, at http://www.who.int/csr/disease/ avian_influenza/guidelines/avian_labtest

16. Uiprasertkul M,Purthavathana P, Sang-sirwut $\mathrm{K}$, et al. Influenza A H5N1 replication sites in humans. Emerg Infect Dis 2005; 11: 1036-1041

17. Govorkova EA, Leneva IA, Goloubeva OG, Bush K and Webster RG. Comparison of efficacies of RWJ-270201, zanamivir, and oseltamivir against $\mathrm{H} 5 \mathrm{~N} 1, \mathrm{H} 9 \mathrm{~N} 2$, and other avian influenza viruses. Antimicrob Agents Chemother 2001; 45: 27232732

18. Moscona A. Neuraminidase inhibitors for influenza. N Eng J Med 2005 Sep 29; 353: $1363-$ 1373.

19. WHO Development of a vaccine effective 
against avian influenza $\mathrm{A}$ (H5N1) infection in humans: update $4 \operatorname{Jan} 20,2004$.

20. Derrick JL, Gomersall CD. Protecting healthcare staff from severe acute respiratory syndrome: filtration capacity of multiple surgical masks. J Hosp Infect 2005; 59: 365-368

21. CDC. Interim recommendations for infection control in health-care facilities caring for patients with known or suspected avian influenza

22. WHO. Influenza $A(H 5 N 1)$ : WHO interim infection control guidelines for health care facilities. Mar 11, 200. Accessed on www.who.int/csr/ disease/avian_influenza/guidelines/infectious/control/ en; December 9, 2005.

23. Hayden FG, Belshe R, Villanueva C, et al. Management of influenza in households: a prospective, randomized comparison of oseltamivir treatment with or without post-exposure prophylaxis. J
Infect Dis 2004; 189: 440- 449.

24. Welliver R, Monto AS, Carewicz O, et al. Effectiveness of oseltamivir in preventing influenza in household contacts: a randomized controlled trial. JAMA 2001; 285:748-754.

25. WHO/INFOSAN. Highly pathogenic $\mathrm{H} 5 \mathrm{~N} 1$ avian influenza outbreaks in poultry and in humans: food safety implications. Nov 4, 2005 Accessed on fao.org/ag/againfo/subjects/documents/en/ fwdsafaty.pdf; December 10, 2005.

26. WHO. Advice to international travelerts. Accessed on www.who.int/cst/disease/avian_influenza/ travel; December 10, 2005.

27. WHO. WHO global influenza preparedness plans: the role of WHO and recommendations for national measures before and during pandemics. Apr 2005. Accessed on www.who.int/csr/disease/ avian_influenza/pandemic/en/; December 10, 2005. 